\title{
Combination of vaping, cannabis and smoking exposure: shorter time to bullous lung disease and pneumothorax
}

\author{
Farhan Ali
}

PGY 2, Internal medicine resident, North Alabama Medical Center, Florence, Alabama, USA

\section{Article Info}

\section{Article Notes}

Received: March 26, 2021

Accepted: April 30, 2021

\section{*Correspondence:}

Dr. Farhan Ali, PGY 2, Internal medicine resident, North Alabama

Medical Center, Florence, Alabama, USA;

Email: drfarhanali30@gmail.com.

(c) 2021 Ali F. This article is distributed under the terms of the Creative Commons Attribution 4.0 International License.

\section{Keywords}

Vaping

Bullous disease

Pneumothorax

Alveolar pathology

\section{Abstract}

We present a 25-year-old Hispanic man with left-sided pleuritic chest pain and dyspnea for three days. His Chest radiograph and $\mathrm{CT}^{\mathrm{A}}$ revealed bilateral apical bullous disease with a $2.5 \mathrm{~cm}$ deep left sided pneumothorax. He was managed with $100 \%$ oxygen via non-rebreather mask and did not require invasive decompression. Patient reported a one-year history of vaping along with tobacco and cannabis smoking exposure from his late teen years. The pneumothorax improved and he was discharged to the pulmonary clinic. The authors review the multiplicative effect of vaping on smoking cannabis and tobacco leading to early apical bullous lung disease. Patients with such significant smoking/vaping history may benefit from prolonged pulmonary and addiction medicine follow-up given the clinical bullous lung disease and risk for continued smoking/vaping. The case emphasizes the potential exponential effect of combining vaping with tobacco and marijuana smoke on lung health leading to disease at extremely young ages.

\section{Introduction}

The prevalence of bullous lung disease among marijuana smokers and vapers is rising especially with the increasing access for vape industry amongst youth ${ }^{1,2}$. The time taken to develop bullous lung disease may be much shorter when a patient has exposure to a variety of sources. Many instances have been reported with young individuals developing pneumothorax due to the use of Marijuana in inhalational form ${ }^{3}$. Herein, we present a case of a young male with a significant history of tobacco and cannabis smoking, as well as vaping who had an event of going through a bullous type pneumothorax.

\section{Case Summary}

This 25-year-old male with no past medical history presented to the ED with left sided pleuritic chest pain for three days; continuous, sharp pain aggravated by deep breaths and exertion but alleviated by rest. He denied fever, chills, cough, or similar illness before. The patient is a security guard with no sick contacts or lifting of heavy weights or recent air-travel but admits to smoking 2-3 wraps of marijuana daily since he was 12 years of age. He has also been vaping weekly for about a year (cannabinoid products per history - unclear about the exact brand and vaporizing carrier oil/ liquid) and smokes tobacco occasionally. Examination revealed a 5'11" tall male with reduced air entry and tympanic percussion notes in the left supraclavicular region. BMI 20.4 and weight 68.5 $\mathrm{kg}$; vitals in normal range. Chest radiograph showed a left sided apical pneumothorax measuring about $2.5 \mathrm{~cm}$ in diameter. He was 


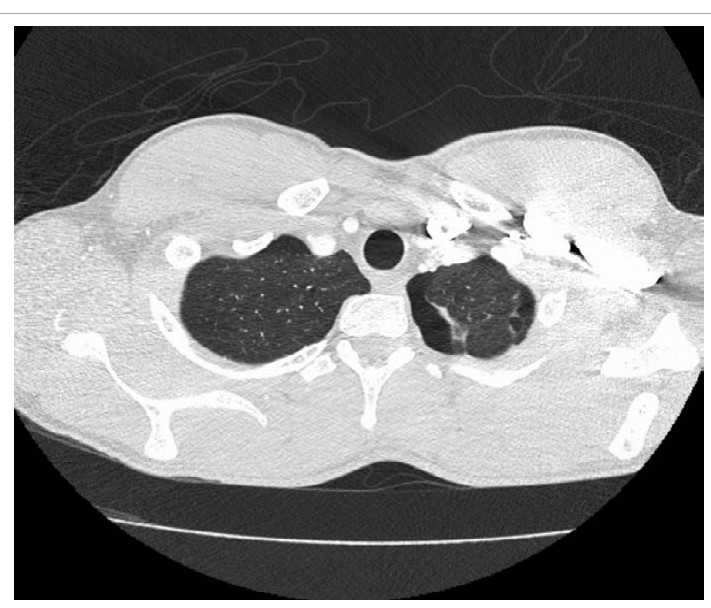

Figure:

started on $100 \%$ oxygen via a non-rebreather mask. CT Chest showed a left apical pneumothorax with paraseptal emphysema and apical bullae more on the right side. Urine toxicology revealed cannabis. Patient was admitted to the ICU due to increase work of breathing and requiring a non-rebreather which may had to be escalated further if needed be. Patient was managed conservatively and did not undergo invasive testing or procedure such as chest tube placement. Follow-up chest x-rays showed no further progression of the pneumothorax and he was discharged within 48 hours to follow with pulmonary outpatient. Chest x-ray as outpatient showed slight improvement of the pneumothorax ${ }^{3}$. He continues to follow with pulmonary \& substance abuse clinic and has quit smoking cannabis, tobacco and vaping. Educational information on smoke exposure, lung injury, addiction behavioral therapy, gutbrain axis and group counseling was shared.

\section{Discussion}

Pneumothorax is a collection of air in the pleural space while spontaneous pneumothorax (SP) is a pneumothorax that occurs without an obvious external cause ${ }^{1}$. There is some overlap in the definition of primary and secondary pneumothorax related to smoking exposure (without versus with clinical lung disease, respectively). The common causes for primary spontaneous pneumothorax are smoking, family history, marfans syndrome, homocystinuria, thoracic endometriosis ${ }^{2}$. The largest case series of spontaneous pneumothorax in patients who were found to have bullous lung disease in the upper lobes was among marijuana smokers ${ }^{4}$. This suggested that marijuana abuse should be listed as a cause of emphysema in young patients $^{4,5}$. Smoking marijuana has been linked with a $66 \%$ larger puff volume and a 33\% rise in breathing depth and inhalation time compared to tobacco ${ }^{6}$. Marijuana smokers tend to hold their breaths about 4 times more than tobacco smokers ${ }^{6,7}$. Newer studies show marijuana can cause lung injury with similar adverse effects like in tobacco users ${ }^{8}$. Smoking tobacco cigarettes could raise the chances of spontaneous pneumothorax by about 22-fold in men and 9-fold in women ${ }^{2,4}$. Cannabis smoking has been linked with SP with a huge burden of carbon monoxide and tar inhalation and accelerated formation of blebs when compared to tobacco users ${ }^{9,10}$. So, tobacco and cannabis users have a higher risk for SP than those smoking one substance ${ }^{10,11}$.

Our patient has been vaping for about a year. Nicholas et al. reported vaping associated spontaneous pneumothorax amongst adolescents a 15-year-old and 16-year-old boy ${ }^{2,9}$. Vaping has now made the headlines with rising morbidity and mortality (e-cig and vape associated acute lung injury-EVALI). The use of electronic cigarettes has rapidly increased over the past decade, particularly amongst adolescents and young adults. About $20.8 \%$ of high school students and $4.8 \%$ of adult's age $18-34$ years are currently vaping (also known as electronic cigarettes) ${ }^{9}$. Most vaping devices are portable, battery-powered with replaceable liquid "pods" and flavors including nicotine ${ }^{2,9}$. The long-term adverse health effects due to vaping are not well known, but a few case reports have been associated with vaping related spontaneous pneumothorax ${ }^{9,12}$. Case reports of EVALI have increased to 2000s in the last few months per CDC and e-cigarette related deaths are rising consistently ${ }^{13}$. What we usually saw at age 50 s with cigarette smoking is now seen in the 20s, thanks to the exponential effect of the combo (vape, cigarette, marijuana). The problem with vaping may not be only from the drug (marijuana, nicotine) but the byproducts of combustion of e-liquid carriers like oils, Vitamin $\mathrm{E}$ (tocopherol), aldehydes, carcinogen particles in the vapor that cause oxidative damage to the lungs ${ }^{14}$. These events of young patients are important in term of Public health as it can lead to better understanding the vaping Industry and help regulate it better. In sum, healthcare providers should educate patients and colleagues about the potential early onset of bullous lung disease with such exposures as well as advocate counseling the very same way it's done for tobacco smoking.

\section{Acknowledgment}

I would like to thank Dr. Nalamatti for her guidance in the initial workup and Dr. K. Chahal for helping in manuscript publication. No financial support was involved in the case report and was an independent write up. The consent was taken from the patient for publishing this case report in various formats.

\section{Conflict of interest}

There were no conflict of interest.

\section{References}

1. Noppen M. Spontaneous pneumothorax: epidemiology, pathophysiology and cause. Eur Respir Rev 2010; 19(117): 217-219. 
2. Nicholas JS, Gwyneth AS, Mary BM, et al. Vaping is a risk factor for spontaneous pneumothorax: Two cases. Journal of Pediatric Surgery Case Reports. 2019; 50: 101305.

3. Mishra R, Patel R, Khaja M. Cannabis-induced bullous lung disease leading to pneumothorax: Case report and literature review. Medicine (Baltimore). 2017; 96(19): e6917. doi: 10.1097/ MD.0000000000006917

4. Beshay M, Kaiser H, Niedhart D, et al. Emphysema and secondary pneumothorax in young adults smoking cannabis. Eur J Cardiothorac Surg. 2007; 32: 834-8.

5. Christopher G, Mathew N. Bleb Point: Mimicker of Pneumothorax in Bullous Lung Disease. West J Emerg Med. 2015; 16(3): 447-449.

6. Rashmi M, Ravi P, Misbahuddin K. Cannabis-induced bullous lung disease leading to pneumothorax Case report and literature review. Medicine. 2017; 96(19): e6917.

7. Ruppert AM, Perrin J, Khalil A, et al. Effect of cannabis and tobacco on emphysema in patients with spontaneous pneumothorax. Diagnostic and Interventional Imaging. 2018; 99: 465-471.

8. Richa S, Rajesh S. Assessment of cases of pneumothorax in a Tertiary Care Hospital - A clinical study. Journal of Advanced Medical and Dental Sciences Research. 2019; 7(8): 183-186.
9. Alex B, Alexander JB, Suliman MA, et al. Recurrent spontaneous pneumothoraces and vaping in an 18-year-old man: a case report and review of the literature. Journal of Medical Case Reports. 2019; 13(1):283

10. Luis IG, Philip W. Effect of cannabis smoking on lung function and respiratory symptoms: a structured literature review. NPJ Primary Care Respiratory Medicine. 2016; 26: 16071.

11. Daniel G, Ryan M. Bilateral spontaneous pneumothoraces with spontaneous pneumomediastinum: An intravenous methamphetamine complication. Respiratory Medicine Case Reports. 2018; 25: 4-5.

12. Sharma M, Anjum H, Bulathsinghala CP, et al. A Case Report of Secondary Spontaneous Pneumothorax Induced by Vape. Cureus. 2019; 11(11): e6067. doi: 10.7759/cureus.6067

13. Outbreak of Lung Illness Associated with Using E-Cigarette Products Investigation Notice. CDC. Accessed: 12 January 2020: https:// www.cdc.gov/tobacco/basic_information/e-cigarettes/severe-lungdisease.html

14. Blount BC, Karwowski MP, Shields PG, et al. Vitamin E acetate in bronchoalveolar-lavage fluid associated with EVALI. New England Journal of Medicine. Epub 2019 Dec 20. 